\title{
On the volatile inventory of Titan from isotopic abundances in nitrogen and methane
}

\author{
Jonathan I. Lunine ${ }^{\mathrm{a}, \mathrm{c}, *}$, Yuk L. Yung ${ }^{\mathrm{b}}$, Ralph D. Lorenz ${ }^{\mathrm{c}}$ \\ ${ }^{a}$ Reparto di Planetologia, CNR-Istituto di Astrofisica Spaziale, Via del Fosso del Cavaliere, 00133, Rome, Italy \\ ${ }^{\mathrm{b}}$ Department of Geological and Planetary Sciences, 170-25 Caltech, Pasadena, CA 91125, USA \\ ${ }^{\mathrm{c}}$ Lunar and Planetary Laboratory, University of Arizona, Tucson, AZ 85721, USA
}

Received 9 November 1998; received in revised form 1 February 1999; accepted 22 February 1999

\begin{abstract}
We analyze recently published nitrogen and hydrogen isotopic data to constrain the initial volatile abundances on Saturn's giant moon Titan. The nitrogen data are interpreted in terms of a model of non-thermal escape processes that lead to enhancement in the heavier isotope. We show that these data do not, in fact, strongly constrain the abundance of nitrogen present in Titan's early atmosphere, and that a wide range of initial atmospheric masses (all larger than the present value) can yield the measured enhancement. The enrichment in deuterated methane is now much better determined than it was when Pinto et al. (1986. Nature 319, 388-390) first proposed a photochemical mechanism to preferentially retain the deuterium. We develop a simple linear theory to provide a more reliable estimate of the relative dissociation rates of normal and deuterated methane. We utilize the improved data and models to compute initial methane reservoirs consistent with the observed enhancement. The result of this analysis agrees with an independent estimate for the initial methane abundance based solely on the present-day rate of photolysis and an assumption of steady state. This consistency in reservoir size is necessary but not sufficient to infer that methane photolysis has proceeded steadily over the age of the solar system to produce large quantities of less volatile organics. Our analysis indicates an epoch of early atmospheric escape of nitrogen, followed by a later addition of methane by outgassing from the interior. The results also suggest that Titan's volatile inventory came in part or largely from a circum-Saturnian disk of material more reducing than the surrounding solar nebula. Many of the ambiguities inherent in the present analysis can be resolved through Cassini-Huygens data and a program of laboratory studies on isotopic and molecular exchange processes. The value of, and interest in, the Cassini-Huygens data can be greatly enhanced if such a program were undertaken prior to the prime phase of the mission. (C) 1999 Elsevier Science Ltd. All rights reserved.
\end{abstract}

\section{Introduction}

The origin of the nitrogen and the methane observed in Titan's atmosphere remains one of the outstanding issues in solar system research almost two decades after the 1980 flyby of Saturn's largest moon by Voyager 1. As is the case for the issue of the origin of nitrogen, water and other volatiles on Earth, the implications of the question extend beyond Titan itself to the solar

\footnotetext{
* Corresponding author. University of Arizona Tucson, Lunar and Planetary Laboratory, 1629 East University Blvd, Tucson, AZ 85721-0092, USA. Tel.: + 1-520-621-2789; fax: + 1-520-621-4933.

E-mail address: jlunine@lpl.arizona.edu (J.I. Lunine)
}

nebula from which the planets were born. Were icy planetesimals principal carriers of volatile molecular species throughout the outer solar system? Did volatilerich icy bodies seed both the inner and outer solar system with organics, water and other molecules, or was there a significant radial confinement of volatilerich solids to the outer solar system? Were environments around the giant planets partly isolated from, and significantly more reducing than, the surrounding solar nebula? What are the implications of the answers to these questions for the physics of the solar nebula itself? These issues, focused on the physics of the outer solar system but also on the delivery of volatiles to both outer solar system solid bodies and the terrestrial planets, were recently reviewed (Lunine et al., 1999). 
A recent first measurement of the ${ }^{15} \mathrm{~N} /{ }^{14} \mathrm{~N}$ ratio in hydrogen cyanide in Titan's atmosphere (Hidayat and Marten, 1998), in concert with improved multiple determinations of the deuterium enrichment in Titan's methane (Gautier, 1997), provide an opportunity to simultaneously estimate the sizes of the initial nitrogen and methane endowments. Because different enrichment processes are more or less important for ${ }^{15} \mathrm{~N} /{ }^{14} \mathrm{~N}$ versus $\mathrm{CH}_{3} \mathrm{D} / \mathrm{CH}_{4}$, it is possible as well to constrain whether the carbon- and nitrogen-bearing volatiles evolved together or through separate reservoirs early in Titan's history. This analysis is aided by a recent measurement of the ${ }^{13} \mathrm{C} /{ }^{12} \mathrm{C}$ ratio in Titan's atmosphere (Hidayat et al., 1997). In turn, these results bear on the nebular issues outlined above; in particular whether Titan's volatiles are products of the particular environment established by Saturn during its formation, or of the solar nebula at large.

Our analysis, based on relatively simple analytic physical models, suggests that the initial nitrogen and methane reservoirs were larger than what is seen today in the atmosphere. While Titan apparently had an epoch of early atmospheric escape, the isotopic evidence suggests that most of the methane was outgassed after this time. Titan's nitrogen and methane were supplied by icy bodies that had a volatile endowment significantly different from that of comets, perhaps originating in a circum-Saturnian disk of material. Our results also suggest a program of laboratory measurements to supplement the data from Cassini-Huygens, and thus provide significant further constraint on the problem. Our presentation begins with a discussion of the isotopic data sets themselves. We then describe the models of atmospheric escape, photochemistry, and consequent isotopic fractionation, along with a justification of particular choices of parameter values and the extent to which they are constrained by laboratory or planetological data. Results of the analysis in terms of initial nitrogen and methane reservoirs follow. In the final section the implications for the origin of Titan's volatile inventory are explored, and recommended or anticipated future measurements in the laboratory and in space are listed.

\section{Isotopic measurements}

\section{1. $H C^{15} N / H C^{14} N$}

The first determination of the ratio of nitrogen isotopes in Titan's atmosphere comes from millimetric wavelength spectroscopic observations of hydrogen cyanide (Marten et al., 1997). Under the assumption that there is no fractionation of the nitrogen isotopic ratio between $\mathrm{HCN}$ and $\mathrm{N}_{2}$, this measurement indicates that the bulk nitrogen is enriched in the heavy

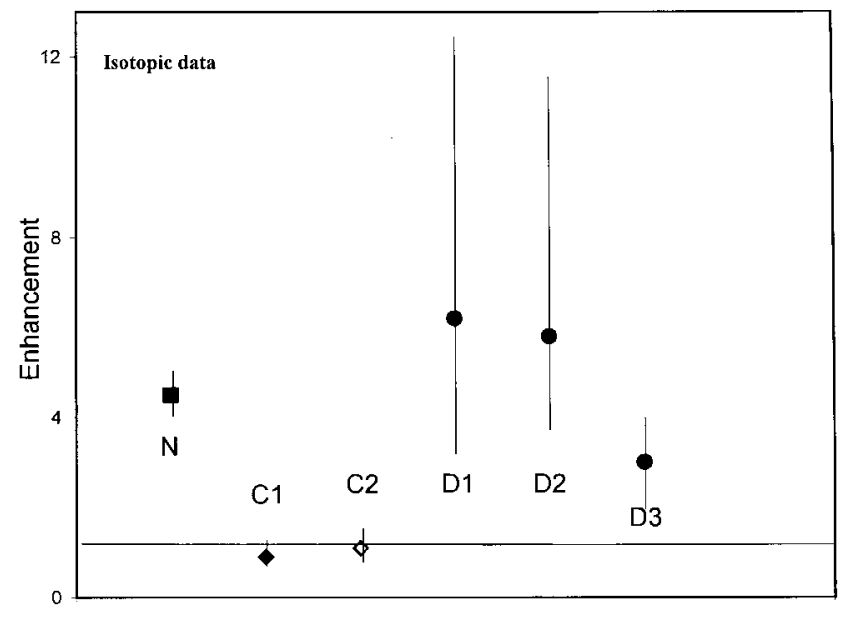

Fig. 1. Summary of the nitrogen (square), carbon (diamonds) and hydrogen (circles) isotopic measurements on Titan considered in this paper, plotted relative to the protosolar (for deuterium) or terrestrial value (for carbon and nitrogen). Key: $\mathrm{N}={ }^{15} \mathrm{~N} /{ }^{14} \mathrm{~N}$ from Hidayat and Marten (1998); D1, 2, 3=D/H from DeBergh et al. (1988), Coustenis et al. (1989), Orton (1992), respectively; $\mathrm{Cl}={ }^{13} \mathrm{C} /{ }^{12} \mathrm{C}$ from $\mathrm{HCN}$ by Hidayat et al. (1997); $\mathrm{C} 2={ }^{13} \mathrm{C} /{ }^{12} \mathrm{C}$ from $\mathrm{C}_{2} \mathrm{H}_{6}$ from Orton (1992). The $\mathrm{C} 2$ data are reported as a range, hence the unfilled diamond indicates no formal centroid value. Horizontal line indicates an enhancement of unity (protosolar or terrestrial value) as a guide to the eye.

isotope ${ }^{15} \mathrm{~N}$ by about 4.5 times relative to the terrestrial value (Hidayat and Marten, 1998); see Fig. 1. Hidayat and Marten (1998) assert further that the terrestrial value is in fact the primordial unfractionated value, and should therefore reflect the material out of which Titan as well as the Earth formed. The arguments underpinning this claim are not detailed in their abstract, but in fact are similar to those offered up in their interpretation of their measured value of $\mathrm{H}^{13} \mathrm{CN} /$ $\mathrm{H}^{12} \mathrm{CN}$. We accept these arguments on their face value, though we note the possibility of separate inner and outer solar system volatile reservoirs that interacted incompletely or negligibly (Lunine et al., 2000).

\section{2. $\mathrm{CH}_{3} \mathrm{D} / \mathrm{CH}_{4}$}

Because Titan's atmosphere has so little hydrogen (a few tenths of a percent in the lower, dense, atmosphere) and methane is so abundant, the $\mathrm{CH}_{3} \mathrm{D} / \mathrm{CH}_{4}$ ratio is the only measurable atmospheric $\mathrm{D} / \mathrm{H}$ ratio. The equivalent $\mathrm{D} / \mathrm{H}$ ratio, for comparison with the protosolar value we adopt here of $2.6( \pm 0.7) \times 10^{-5}$ (Geiss and Gloeckler, 1998; Mahaffy et al., 1998) is just one-fourth the $\mathrm{CH}_{3} \mathrm{D} / \mathrm{CH}_{4}$ ratio. Early determinations of $\mathrm{CH}_{3} \mathrm{D} / \mathrm{CH}_{4}$ had large error bars and suggested a significant enrichment over the protosolar value (Gautier, 1997). De Bergh et al. (1988) found an equivalent $\mathrm{D} / \mathrm{H}$ of $16.1(+16.5,-8) \times 10^{-5}$ from ground-based data; Coustenis et al. (1989) derived D/ $\mathrm{H}=15.0(+15.0,-10.0) \times 10^{-5}$ from Voyager spectra. 
However, a somewhat more recent determination from IRTF measurements at 8.7 micron wavelength gives a smaller value, $\mathrm{D} / \mathrm{H}=7.75 \pm 2.25 \times 10^{-5}$, equivalent to 2 to 5 times the protosolar value (Orton, 1992). Lecluse et al. (1996) argue that this more precise determination supersedes the earlier measurements. While we do not have any independent assessment of the quality of the data, we adopt this point of view and hence emphasize the most recent determination of Titan's $\mathrm{CH}_{3} \mathrm{D} / \mathrm{CH}_{4}$.

\section{3. $\mathrm{H}^{13} \mathrm{CN} / \mathrm{H}^{12} \mathrm{CN}$}

The ratio of heavy to light carbon in Titan's atmosphere was determined in $\mathrm{HCN}$ through millimeter measurements at $88.63 \mathrm{GHz}$ by Hidayat et al. (1997), who do not provide formal error bars but show instead their spectra to be consistent with $0.0083<{ }^{13} \mathrm{C} /{ }^{12} \mathrm{C}<0.014$. (It is standard to report the ratio ${ }^{12} \mathrm{C} /{ }^{13} \mathrm{C}$, but because we are dealing with the ratio of the heavy to light isotope for deuterium and nitrogen we find it less confusing to invert the ratio and its reported value in the interest of consistency.) The result may be compared with the carbon isotopic ratio in Titan's ethane determined from 12 micron wavelength spectra: ${ }^{12} \mathrm{C}^{13} \mathrm{CH}_{6} /{ }^{12} \mathrm{C}^{12} \mathrm{CH}_{6}=0.025 \pm 0.005$ (Orton, 1992). Since there are two carbon atoms per molecule this corresponds to $0.010<{ }^{13} \mathrm{C} /{ }^{12} \mathrm{C}<0.015$, consistent with the $\mathrm{HCN}$ determination (Fig. 1).

Hidayat et al. (1997) make two assertions regarding the carbon isotopic ratio. The first is that the range of the values derived for Titan is consistent with the terrestrial value. The second is that the ratio should be similar throughout the solar system and reflect primarily the degree of thermonuclear evolution of the material from which the Sun and planets formed. The first of these is certainly reasonable, as the terrestrial (really, meteoritical) value is around 0.011 (Van Dishoeck et al., 1993). However, the second claim is problematical given that grains in Comet Halley, measured directly from the Giotto and Vega spacecraft, have ${ }^{13} \mathrm{C} /{ }^{12} \mathrm{C}$ ranging from 0.0002 to 1 (Mumma, 1997). Bulk ${ }^{13} \mathrm{C} /{ }^{12} \mathrm{C}$ values in the outer solar system range from 0.006 to 0.2 (Fegley, 1997). In any event, there is no reason to presume inner and outer solar system volatiles were well mixed with each other.

Given these uncertainties, we must approach with caution the conclusion that Titan's ${ }^{13} \mathrm{C} /{ }^{12} \mathrm{C}$ value reflects a primordial, unfractionated carbon budget. However, the Hidayat et al. (1997) conclusions are the simplest ones consistent with the available data. This is especially the case given the difficult conditions under which the Halley results were obtained, and the fact that separate, minor phases depleted in ${ }^{13} \mathrm{C} /{ }^{12} \mathrm{C}$ are found in meteorites and recognized to reflect particular nucleosynthetic environments (Ott, 1993). We therefore accept their conclusion that the carbon isotopic ratio is terrestrial.

\section{Calculations}

\subsection{Enrichment of heavy nitrogen through escape}

The nitrogen in Titan's atmosphere is assumed to be escaping at the exobase in atomic form. Below the exobase and above the homopause diffusive separation of species according to atomic weight occurs. Thus the ratio ${ }^{15} \mathrm{~N} /{ }^{14} \mathrm{~N}$ progressively decreases from the homopause upwards to the exobase, so that the composition of escaping gas is depleted in ${ }^{15} \mathrm{~N}$ relative to the bulk atmospheric value. Hence over time the atmosphere becomes progressively enriched in the heavier isotope of nitrogen, independent of any effects associated with kinetic energy partitioning during photodissociation or charged particle dissociation of $\mathrm{N}_{2}$. These latter effects create additional fractionation favoring retention of ${ }^{15} \mathrm{~N}$, and are discussed below.

Diffusively generated fractionation becomes more efficient as the separation between the homopause and exobase altitudes increases, but weakens as the altitudes of both levels increase (because the gravitational acceleration declines). As atmospheric escape progresses toward blowoff, the level at which atoms escape moves below the exobase into the bulk atmosphere; this in turn eliminates the diffusive fractionation according to atomic or molecular weight. The presentday Titan atmosphere is not close to the blowoff regime, though some models of a thick primordial Titan atmosphere invoke blowoff to arrive at the present atmospheric mass (Lunine, 1985). In what follows we will consider Titan's early atmosphere to be in a regime, with respect to escape, similar to that in the present-day. We will then consider the consistency of this assumption with the results derived from such a model.

Considering mass fractionation during escape to be a Rayleigh process, the ratio of the abundance of the heavier gas $n_{2}$ to the lighter gas $n_{1}$ is

$\frac{n_{2}}{n_{1}}=(1+y) \frac{\mathrm{d} n_{2}}{\mathrm{~d} n_{1}}$

where $y$ is a fractionation factor. For non-thermal escape involving mass-driven diffusive separation the fractionation factor is (Hunten et al., 1989):

$y=\exp \left(\Delta z / H_{\mathrm{d}}\right)-1$.

The distance from homopause to exobase is $\Delta z$, and $H_{\mathrm{d}}$ is a diffusional scale height 


$$
H_{\mathrm{d}}=\frac{k T}{\left(m_{2}-m_{1}\right) g}
$$

where $k$ is Boltzmann's constant, $T$ temperature, $m_{x}$ the atomic mass of isotope $x$, and $g$ the gravitational acceleration. Although $T$ and $g$ vary with altitude, $T$ does so weakly (see below). The gravitational acceleration is sensitive to the radial distance from Titan's center: by choosing a radius halfway between the exobase and the homopause we fix $g$ and thereby integrate Eq. (1) analytically to find

$$
\frac{n_{1}^{0}}{n_{1}}=\left(\frac{n_{2}}{n_{1}} / \frac{n_{2}^{0}}{n_{1}^{0}}\right)^{(1+y) / y}
$$

where $n_{x}^{0}$ indicates the initial amount of isotope $x$. For nitrogen, Hidayat and Marten (1998) measured the ratio ${ }^{15} \mathrm{~N} /{ }^{14} \mathrm{~N} \equiv n_{2} / n_{1}$; the initial value $n_{2}^{0} / n_{1}^{0}$ prior to atmospheric enrichment is assumed to be the terrestrial value as in the analysis of Hidayat and Marten and others to date. Then using Eqs. (2)-(4) we solve for $n_{1}^{0} / n_{1}$, the ratio of the initial nitrogen abundance to the present value. This is the original atmospheric mass relative to that at present, assuming $\mathrm{N}_{2}$ was the dominant species then as today.

The computation of $y$ presented above assumes that fractionation is achieved solely through the action of diffusive separation. However, impact (electron) ionization and dissociative recombination of nitrogen partition the kinetic energy between the isotopes such that the velocity distributions of the lighter and heavier isotopes are different. On Mars, while dissociative recombination produces some light nitrogen atoms with sufficient velocity for escape, the heavy nitrogen is almost always left behind in the atmosphere (Fox and Hac, 1997; Lammer and Bauer, 1991). This in turn provides an additional fractionation effect that works in the same direction as the diffusive separation, enhancing the atmospheric abundance of the heavier isotope with time. The diffusive $\left(y_{\mathrm{f}}\right)$ and dissociative $\left(y_{\mathrm{d}}\right)$ mass fractionations can be computed independently and then combined so that Eq. (4) becomes

$$
\frac{n_{1}^{0}}{n_{1}}=\left(\frac{n_{2}}{n_{1}} / \frac{n_{2}^{0}}{n_{1}^{0}}\right)^{\psi} ; \quad \psi=\frac{\left(1+y_{\mathrm{f}}\right)\left(1+y_{\mathrm{d}}\right)}{\left(1+y_{\mathrm{f}}\right)\left(1+y_{\mathrm{d}}\right)-1} .
$$

The computation of $y_{\mathrm{d}}$ is not straightforward. Fox and Hac (1997) find $y_{\mathrm{d}}$ ranging from 0.5 to 0.6 in the Martian atmosphere, where impact dissociation dominates the loss of nitrogen. They find this process to over-fractionate the nitrogen isotopes so that ${ }^{15} \mathrm{~N} /{ }^{14} \mathrm{~N}$ is larger than the present measured value for an initial value assumed to be that of the Earth's. They therefore invoke a scenario in which the nitrogen is embedded in a much more massive atmosphere in which escape is presumed to occur below the homopause, rendering fractionation much less efficient. It is also possible that the escape efficiency of the charged particle dissociative processes has been overestimated. In fact, for Titan it must be less efficient than for Mars because of Titan's much lower gravity at the altitude of escape, which reduces the importance of the mass difference between light and heavy isotopes. The escape energies for nitrogen atoms (both ${ }^{14} \mathrm{~N}$ and ${ }^{15} \mathrm{~N}$ ) from the exobase of Titan lie well below the mean energy obtained in laboratory fragmentation of $\mathrm{N}_{2}$ by electron impact (Cosby, 1993), suggesting little selectivity for escape from Titan by this process. Complicating the problem further is that atmospheric sputtering of nitrogen atoms at and above the exobase may be the principal loss mechanism for the present atmosphere of Titan (Lammer and Bauer, 1993). So we expect $y_{\mathrm{d}}$ for Titan to be lower than that for Mars (perhaps close to 0), but to illustrate the effect we run fractionation calculations for several different values of $y_{\mathrm{d}}$ within the range 0 to 0.6 .

In the calculation presented below we choose a nominal exobase radius of $4175 \mathrm{~km}$, as measured from the center of Titan (and corresponding to a height above the surface of $1600 \mathrm{~km}$ ) (Strobel et al., 1992). However, a denser early atmosphere on Titan, the selfconsistent result of the present calculations, would have a higher exobase. To explore the sensitivity of our analysis to the choice of exobase level we also do calculations with an exobase radius of $4500 \mathrm{~km}$. The homopause altitude has been constrained from Voyager ultraviolet spectrometer profiles of methane, along with other data, to lie at 985 to $1125 \mathrm{~km}$ altitude, equivalent to radii of 3560 to $3700 \mathrm{~km}$ (Strobel et al., 1992; Vervack, 1997). Earlier authors computed homopause levels as low as $3200 \mathrm{~km}$ from Titan's center based on photochemical models (Yung et al., 1984). Hence we parameterize our results in terms of a homopause situated between 3200 and $3700 \mathrm{~km}$, but prefer values above $3550 \mathrm{~km}$. The temperature profile between the homopause and exobase is a fit to Strobel et al. (1992). The temperature is $175 \mathrm{~K}$ above $3500 \mathrm{~km}$ and declines linearly to $140 \mathrm{~K}$ at $3200 \mathrm{~K}$. Moderating the temperature decline, such that at $3200 \mathrm{~km}$ the temperature is $150 \mathrm{~K}$, does not change the results described below to any significant extent.

The measured ratio of $\mathrm{HC}^{15} \mathrm{~N} / \mathrm{HC}^{14} \mathrm{~N}$ in Titan's atmosphere is assumed to reflect an enrichment in heavy nitrogen by a factor of 4.5 over the putative primordial value based on the terrestrial atmosphere (Hidayat and Marten, 1998). As noted above, we accept this line of reasoning with the caution that there may have been different inner and outer solar system isotopic abundances and different gaseous and condensed reservoirs supplying various bodies. Further, we raise again the caveat that initial abundance determinations in inherently noisy spectra have in the past led to values that 
ultimately are lowered by subsequent data or reanalysis of the original data set. While we do not challenge the analysis of Hidayat and Marten (1998), and we applaud their work, we feel it appropriate to consider the possibility of a factor of two smaller enhancement in ${ }^{15} \mathrm{~N} /{ }^{14} \mathrm{~N}$. Thus we pick isotopic enhancements between two and four.

\subsection{Enrichment of deuterium through methane photochemistry}

The photochemical enrichment model follows the formalism of Pinto et al. (1986). The system is closed with respect to carbon but the dissociated hydrogen (either deuterium or protium) is lost to space. The reservoir may be all in the atmosphere, or reside partly in gaseous and condensed states. The condensed phase (liquid seas of hydrocarbons, or methane trapped in porous crustal structures) remains cold enough to avoid isotopic re-equilibration among the methane and other hydrocarbon species (e.g., $\mathrm{H}+\mathrm{CH}_{3} \mathrm{D} \rightarrow \mathrm{HD}+$ $\mathrm{CH}_{3}$ is too slow to be significant). Also the flux of cosmic rays at the surface is sufficiently small that cosmic-ray driven fractionation is a minor effect (Sagan and Thompson, 1984).

Define $A(t) \equiv$ total abundance of methane in the system at time $t$ and $B(t) \equiv$ total abundance of deuterated methane at time $t$. Then photolytic destruction relates the abundance at time 0 to that at time $t$ as

$A(t)=A(0) \exp \left(-\lambda_{A} t\right)$

$$
B(t)=B(0) \exp \left(-\lambda_{B} t\right)
$$

where because of the significantly higher binding energy of the $\mathrm{C}-\mathrm{D}$ bond compared to the $\mathrm{C}-\mathrm{H}$ bond the rate $\lambda_{B}=q \lambda_{A}, 0.75 \leq q<1$. Note that $q=0.75$ is a physical lower limit implying that the deuterium atom in $\mathrm{CH}_{3} \mathrm{D}$ is never removed by photolysis. (Strictly speaking slightly smaller values are possible because a small amount of $\mathrm{CH}_{2} \mathrm{D}_{2}$ and more heavily deuterated methane exist in the atmosphere; but the effect is too small to be worth considering.) Now define the ratio of the deuterated to non-deuterated methane at time $t$ to be $f(t)=B(t) / A(t)$, the ratio of the methane reservoir size at time zero to the present value as $R=A(0) / A(t)$, and the enrichment over time in deuterium due to atmospheric photochemistry to be $E=f(t) / f(0)$. An analytic relationship exists between $E$ and $R$ (Pinto et al., 1986):

$R=E^{1 /(1-q)}$.

Thus, there is a direct relationship between the enhancement over the primordial value of deuterated methane (relative to non-deuterated) in the present surface-atmosphere system, and the size of the original reservoir of methane (surface plus atmosphere) relative to that at present.

To apply this model usefully to the enrichment of $\mathrm{CH}_{3} \mathrm{D}$ in Titan's atmosphere we compute tighter constraints on the value of $q$ than are given by the simple considerations above. As pointed out in Pinto et al. (1986) the most important isotopic fractionation of $\mathrm{CH}_{4}$ as it is destroyed photochemically depends on the relative rates of the two abstraction reactions

$\mathrm{C}_{2} \mathrm{H}+\mathrm{CH}_{4} \rightarrow \mathrm{C}_{2} \mathrm{H}_{2}+\mathrm{CH}_{3}$

$\mathrm{C}_{2} \mathrm{H}+\mathrm{CH}_{3} \mathrm{D} \rightarrow \mathrm{C}_{2} \mathrm{H}_{2}+\mathrm{CH}_{2} \mathrm{D}$

$\rightarrow \mathrm{C}_{2} \mathrm{HD}+\mathrm{CH}_{3}$.

Let $k_{2}=k_{2 \mathrm{a}}+k_{2 \mathrm{~b}}$. The fractionation depends on the ratio $q=k_{2} / k_{1}$. This ratio has never been measured. Pinto et al. (1986) adopted the value $q=0.75$ for their model $\mathrm{A}$, and a higher value of 0.875 for model $\mathrm{B}$. We argue here that on the basis of recent advances in the chemical kinetics of deuterated species that in fact $0.8 \leq q \leq 0.88$.

In the absence of a laboratory measurement for the ratio $q$, we resort to an estimate using analogous reactions. Recently Gierczak et al. (1997) studied the abstraction reactions between $\mathrm{OH}$ and the deuterated isotopomers

$\mathrm{OH}+\mathrm{CH}_{4-n} \mathrm{D}_{n} \rightarrow$ products

where $n$ varies from 0 to 4 , with $\mathrm{CH}_{4}$ represented by $n=0$ and $\mathrm{CD}_{4}$ represented by $n=4$. The rate constants for the five isotopomers are summarized in Table 1. The measurements are most accurate at room temperature $(300 \mathrm{~K})$. The values relative to $\mathrm{CH}_{4}$ at this temperature are given by the quantity $R_{1}$. Note that $R_{1}$ varies smoothly from 1 to 0.14 as $n$ varies from 0 to 4 , suggesting a simple linear theory is valid. Suppose the rate constants shown in Table 1 are com-

Table 1

Rate constants for $\mathrm{OH}+\mathrm{CH}_{4}$ and isotopomers ${ }^{\mathrm{a}}$

\begin{tabular}{lllllc}
\hline$n$ & Reaction & $\begin{array}{l}k(T) \\
\mathrm{cm}^{3} \mathrm{~s}^{-1}\end{array}$ & $\begin{array}{l}k(300 \mathrm{~K}) \\
\mathrm{cm}^{3} \mathrm{~s}^{-1}\end{array}$ & $R_{1}{ }^{\mathrm{b}}$ & $R_{1}{ }^{\mathrm{c}}$ \\
\hline 0 & $\mathrm{OH}+\mathrm{CH}_{4}$ & $1.88 \times 10^{-12} \mathrm{e}^{-1695 / T}$ & $6.61 \times 10^{-15}$ & 1 & $(1)$ \\
1 & $\mathrm{OH}+\mathrm{CH}_{3} \mathrm{D}$ & $3.11 \times 10^{-12} \mathrm{e}^{-1910 / T}$ & $5.34 \times 10^{-15}$ & 0.81 & 0.79 \\
2 & $\mathrm{OH}+\mathrm{CH}_{2} \mathrm{D}_{2}$ & $2.30 \times 10^{-12} \mathrm{e}^{-1930 / T}$ & $3.70 \times 10^{-15}$ & 0.56 & 0.59 \\
3 & $\mathrm{OH}+\mathrm{CHD}_{3}$ & $1.46 \times 10^{-12} \mathrm{e}^{-1970 / T}$ & $2.05 \times 10^{-15}$ & 0.31 & 0.29 \\
4 & $\mathrm{OH}+\mathrm{CD}_{4}$ & $1.00 \times 10^{-12} \mathrm{e}^{-2100 / T}$ & $9.12 \times 10^{-16}$ & 0.14 & $(0.14)$ \\
\hline
\end{tabular}

\footnotetext{
${ }^{a}$ Measurements by Gierczak et al. (1997).

${ }^{\mathrm{b}}$ Rate constant measured at $300 \mathrm{~K}$ relative to $\mathrm{OH}+\mathrm{CH}_{4}$.

${ }^{\mathrm{c}}$ Rate constant at $300 \mathrm{~K}$ relative to $\mathrm{OH}+\mathrm{CH}_{4}$ from linear theory; ( ) = fixed at the measured value.
} 
Table 2

Rate constants for $\mathrm{Cl}+\mathrm{CH}_{4}$ and isotopomers at $295 \mathrm{~K}^{\mathrm{a}}$

\begin{tabular}{llllc}
\hline$n$ & Reaction & $k \mathrm{~cm}^{3} \mathrm{~s}^{-1}$ & $R_{2}{ }^{\mathrm{b}}$ & $R_{2}{ }^{\mathrm{c}}$ \\
\hline 0 & $\mathrm{Cl}+\mathrm{CH}_{4}$ & $9.56 \times 10^{-14}$ & 1 & $(1)$ \\
1 & $\mathrm{Cl}+\mathrm{CH}_{3} \mathrm{D}$ & $7.35 \times 10^{-14}$ & 0.77 & 0.77 \\
2 & $\mathrm{Cl}+\mathrm{CH}_{2} \mathrm{D}_{2}$ & $4.57 \times 10^{-14}$ & 0.48 & 0.52 \\
3 & $\mathrm{Cl}+\mathrm{CHD}_{3}$ & $2.32 \times 10^{-14}$ & 0.24 & 0.25 \\
4 & $\mathrm{Cl}+\mathrm{CD}_{4}$ & $6.10 \times 10^{-15}$ & 0.064 & $(0.064)$ \\
\hline
\end{tabular}

a All measurements from Wallington and Hurley (1992), except for $\mathrm{Cl}+\mathrm{CH}_{4}$ from DeMore et al. (1997).

${ }^{\mathrm{b}}$ Measured rate constant relative to $\mathrm{Cl}+\mathrm{CH}_{4}$.

${ }^{\mathrm{c}}$ Rate constant relative to $\mathrm{Cl}+\mathrm{CH}_{4}$ as deduced from linear theory; ( ) = fixed at the measured value.

posed of the superposition of individual $\mathrm{C}-\mathrm{H}$ or $\mathrm{C}-\mathrm{D}$ bond-breaking sub-rate constants, i.e., each of the above reactions is given by

$k=(4-n) k_{\mathrm{C}-\mathrm{H}}+n k_{\mathrm{C}-\mathrm{D}}$.

From Table 1 we have $k_{\mathrm{C}-\mathrm{H}}=0.25, k_{\mathrm{C}-\mathrm{D}}=0.035$. This linear theory predicts $R_{1}=0.79,0.59$, and 0.29 for $n=1,2,3$, respectively. These values are in excellent agreement with the measured ratios $R_{1}=0.81,0.56$, and 0.31 , respectively.

Further support for this linear theory is obtained by examining the abstraction reactions between $\mathrm{Cl}$ and $\mathrm{CH}_{4}$ as summarized in Table 2 (Wallington and Hurley, 1992) and DeMore et al. (1997). Again there is a nearly linear dependence of the relative rate constants $R_{2}$ on $n$. In this case the linear theory predicts $R_{2}=0.77,0.52$, and 0.25 , as compared to the measured ratios $R_{2}=0.77,0.48,0.24$. The agreement is also very good. Comparing Tables 1 and 2, we note the success of the simple theory is remarkable as the absolute rate constants between the reactions with $\mathrm{OH}$ and $\mathrm{Cl}$ differ by more than an order of magnitude.

The linear theory suggests that we can achieve a reasonable estimate for $q$ if the rate constant for the following reaction is measured

$\mathrm{C}_{2} \mathrm{H}+\mathrm{CD}_{4} \rightarrow \mathrm{C}_{2} \mathrm{HD}+\mathrm{CD}_{3}$.

Let $r_{3}=k_{3} / k_{1}$. The above reasoning suggests that a good estimate for $q$ is given by

$q=0.75+0.25 r_{3}$.

The rate constant $k_{1}$ has been measured at room temperature to be in the range 1.3 to $4.8 \times 10^{-12} \mathrm{~cm}^{3} \mathrm{~s}^{-1}$ (Laufer, 1981; Renlund et al., 1982; Lander et al., 1990). The higher values are probably caused by contamination from vibrationally or electronically excited states of the ethynyl radical produced in laser photolysis. Thus the lower value range $1.3 \times 10^{-12} \mathrm{~cm}^{3} \mathrm{~s}^{-1}$ from Laufer (1981) is more appropriate for atmospheric modeling. Most of the destruction of $\mathrm{CH}_{4}$ on
Titan takes place in the stratosphere where the mean temperature is around $175 \mathrm{~K}$. If we adopt the lower value for $k_{1}$, an estimate for its temperature dependence yields

$k_{1}=2.8 \times 10^{-12} \mathrm{e}^{-250 / T} \mathrm{~cm}^{3} \mathrm{~s}^{-1}$.

At $175 \mathrm{~K}, k_{1}=6.7 \times 10^{-13} \mathrm{~cm}^{3} \mathrm{~s}^{-1}$, a value that is within an order of magnitude of the rate constant for $\mathrm{Cl}+\mathrm{CH}_{4}$ at room temperature. This implies that we may use the values of $R_{1}(n=4)$ and $R_{2}(n=4)$ to estimate $r_{3}$. However, as $k_{1}$ is faster than both the $\mathrm{Cl}$ and $\mathrm{OH}$ reactions, we expect $r_{3}$ to be somewhat larger. In this estimate we assume that $r_{3}=0.2$, yielding a value for $q=0.80$ as the lower bound. An upper bound can be made on the basis of the abstraction reactions

$\mathrm{C}_{2} \mathrm{H}+\mathrm{H}_{2} \rightarrow \mathrm{C}_{2} \mathrm{H}_{2}+\mathrm{H}$

$\mathrm{C}_{2} \mathrm{H}+\mathrm{D}_{2} \rightarrow \mathrm{C}_{2} \mathrm{H}_{2}+\mathrm{D}$.

The ratio $k_{3} / k_{5}$ has been measured to be 0.52 at room temperature (Lander et al., 1990). Adopting this value for $r_{3}$ yields $q=0.88$.

Hence, in the twelve years since Pinto et al. (1986) made the initial conjecture that $0.75 \leq q \leq 0.875$, considerable progress has been made in chemical kinetics that provides circumstantial evidence supporting this general range. We have tightened the limits somewhat to be between 0.80 and 0.88 . However, the most significant result we have obtained is the simple linear theory connecting the rate constant of $\mathrm{CD}_{4}$ to that of $\mathrm{CH}_{3} \mathrm{D}$. The former is much easier to measure in the laboratory than the latter.

The total methane abundance in the atmosphere of Titan is known to within a factor of 4 from Voyager data (Courtin et al., 1995). However, the amount of methane in thermodynamic contact with the atmosphere, either on the surface or accessible in a near-surface water-ice crust, is very poorly constrained. The current atmospheric methane abundance could not be increased by more than a factor of 5 to 10 without creating global saturation and hence the presence of liquid methane on the surface. Numerous remote sensing data (e.g., Smith et al., 1996) rule out global coverage of methane-bearing liquids, but many times the atmospheric mass of methane could be hidden in highlatitude crater lakes (Lorenz and Lunine, 1997) or subsurface reservoirs (Stevenson, 1992). The model considered here for photochemical enrichment of methane is consistent with the assumption that methane photochemistry has proceeded for a significant fraction of the history of Titan, which in turn requires resupplying the atmosphere many time over with methane. This, however, is a result of the analysis detailed below, rather than an ad hoc assumption. 


\section{Results}

\subsection{Nitrogen}

Fig. 2 plots the ratio of the initial to present nitrogen abundance as a function of the homopause level, for the low and high exobase cases, and the nominal measured ${ }^{15} \mathrm{~N} /{ }^{14} \mathrm{~N}$ value. We also show a curve assuming the measured ${ }^{15} \mathrm{~N} /{ }^{14} \mathrm{~N}$ is only twice the 'primordial' value for the reasons discussed in the previous section. In this figure only fractionation caused by diffusive separation is considered. The results illustrate an important point: the high measured value of heavy nitrogen in Titan's atmosphere implies that the early atmosphere was more massive than the present one. For a fixed exobase, the atmospheric mass that must be lost to fractionate the required amount of nitrogen rises as the homopause level rises, because the efficiency of diffusive separation decreases as $\Delta z$ shrinks [Eq. (2)]. For a low homopause level of $3200 \mathrm{~km}$ radial distance, the initial nitrogen abundance is approximately 30 times the present atmospheric pressure. If we accept a factor of two lower value of the required isotopic enhancement the initial atmosphere need be only 5 times as massive as the present one. For a homopause value of $3600 \mathrm{~km}$ the required initial reservoir of nitrogen ranges from 30 to as much as 700 times the current atmosphere.

Fig. 3 displays the effect of fractionation from nitrogen dissociation on the ratio of the initial to present atmospheric mass on Titan. The baseline case is that of the solid line in Fig 2: the observed ${ }^{15} \mathrm{~N} /{ }^{14} \mathrm{~N}$ value is four times the primordial one and the exobase is at $4175 \mathrm{~km}$ from Titan's center. The four lines correspond to values of $y_{\mathrm{d}}$ ranging from 0 to 0.6 . The higher values of $y_{\mathrm{d}}$ have a significant effect on the fractionation, lowering the required initial inventory and rendering it much less sensitive to the value of $\Delta z$. For $y_{\mathrm{d}}=0.6$ initial reservoirs range from 9 to 18 times the present atmospheric mass; for $y_{\mathrm{d}}=0.4$ the range is 11 to 30 . However, given the likelihood that $y_{\mathrm{d}}$ is small, even zero, it may not be plausible to invoke this mechanism to enhance the fractionation.

Initial nitrogen reservoirs hundreds of times the current atmospheric mass, if entirely in the vapor phase, imply very massive primordial atmospheres. Lunine (1985) argued that gas-rich accretion of Titan in a circum-Saturnian nebula could produce a primordial at-

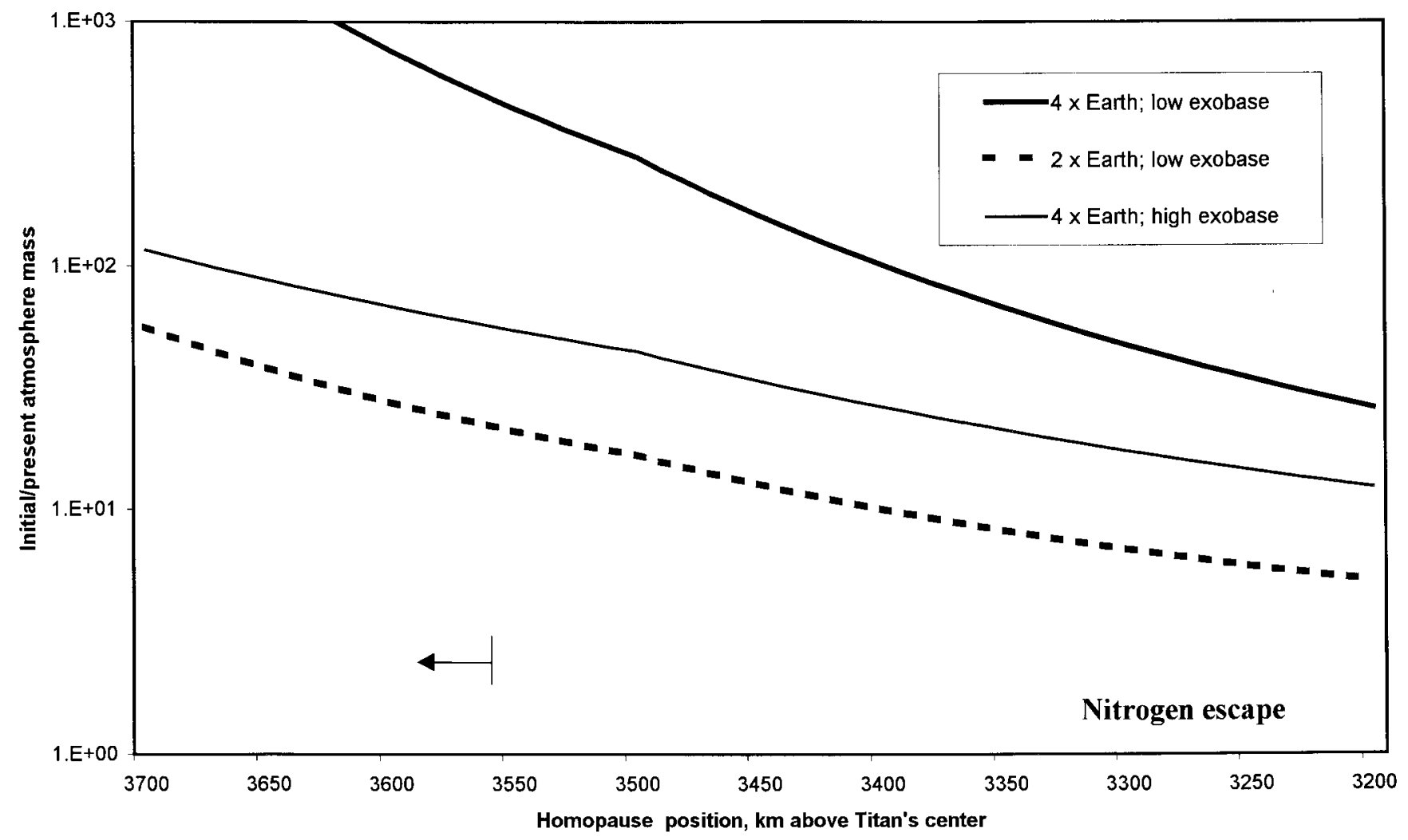

Fig. 2. Isotopic fractionation caused by escape of nitrogen. Size of Titan's initial nitrogen reservoir, normalized to the present nitrogen atmospheric mass, as a function of the homopause distance from Titan's center. The three curves express different choices for the measured nitrogen isotope fractionation (twice and four times primordial) and exobase height (4175 and $4500 \mathrm{~km}$ above the center of Titan). Arrow demarcates lower limit of preferred homopause height based on Voyager ultraviolet observations (see text). 


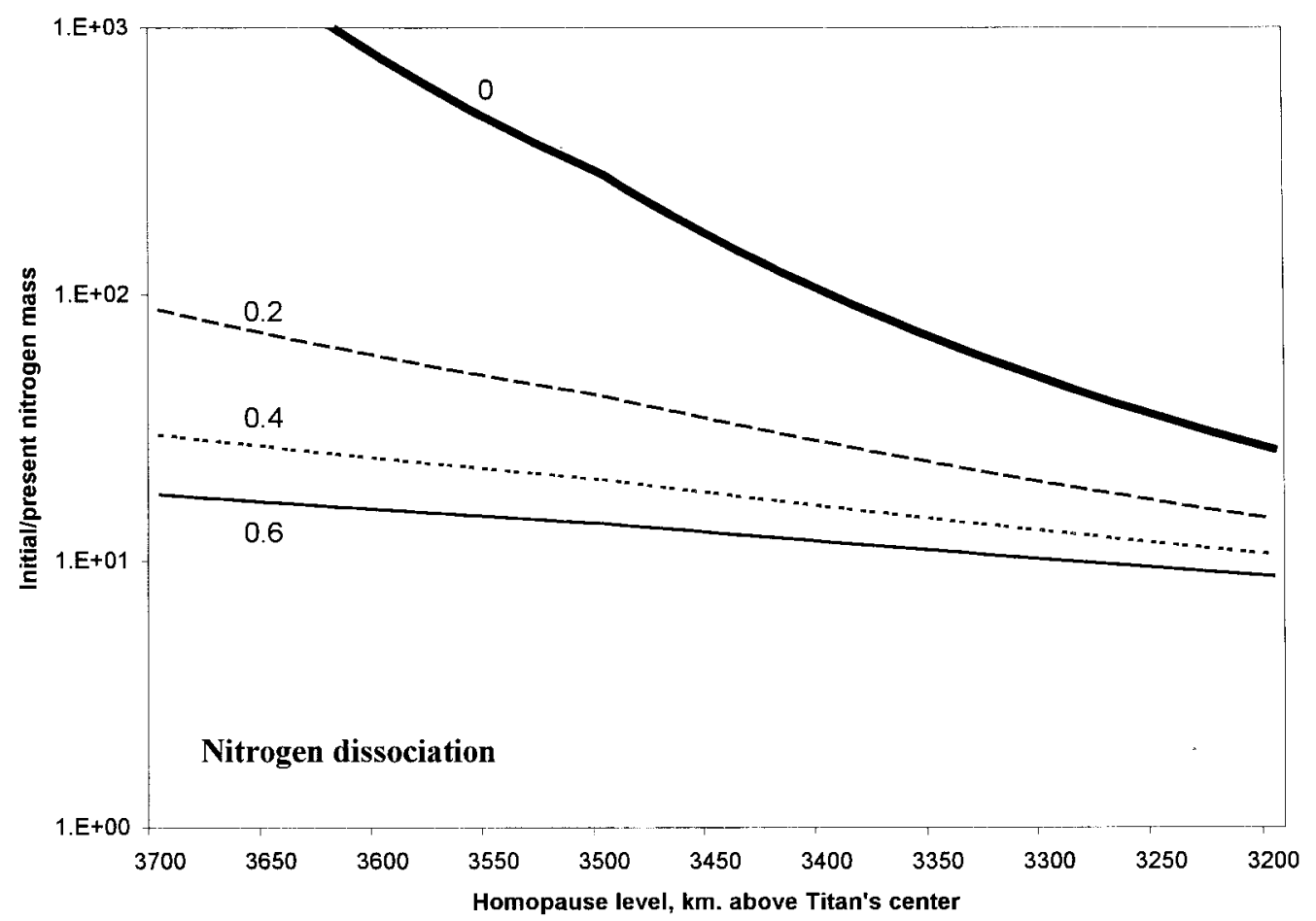

Fig. 3. Effective of nitrogen dissociation on the escape calculations. Plotted as in Fig. 2 is the size of the initial nitrogen reservoir versus homopause distance. The four curves show the effect of differing fractionation factors $y_{d}$ caused by dissociative recombination and impact ionization of nitrogen, as described in the text. The baseline model is the heavy isotope fraction 4 times primordial, with an exobase height of $4175 \mathrm{~km}$, from Fig. 2.

mosphere of hundreds of bars in pressure. Such an atmosphere would have to be lost by blowoff early in Titan's history, and blowoff greatly decreases the efficiency of the isotopic fractionation compared to that computed here. Indeed, the thick atmosphere case seems internally inconsistent for that reason alone, and suggests one of the following: efficient fractionation mechanisms operated in a more modest initial atmosphere leading to the observed nitrogen isotopes, for example, via the high exobase case considered in Fig. 2. Alternatively, the observed nitrogen isotopic ratio is closer to the primordial value than inferred by Hidayat and Marten (1998), perhaps by a factor of 2 as considered also in Fig. 2.

\subsection{Methane}

Fig. 4 summarizes the results for deuterium enrichment via photodissociation. It plots the initial methane reservoir (normalized to the present one) against $q$, the relative dissociation rates of $\mathrm{CH}_{3} \mathrm{D}$ and $\mathrm{CH}_{4}$. Four cases are selected, corresponding to a range in both the measured ratio of $\mathrm{CH}_{3} \mathrm{D}$ and $\mathrm{CH}_{4}$ and the primordial value. For the latter we assume at least modest enrichment in the material out of which Titan formed: 1.5 times the protosolar value as a baseline, and one case computed with twice that enrichment. Since the curves depend directly on the ratio of the present to primordial deuterium enrichments, they can simply be scaled for other values of the primordial enrichment. A smaller value of $q$ yields greater fractionation for a given amount of methane photolysis, because it corresponds to deuterium being more tightly bound. Hence, the smaller $q$ is the smaller is the required initial methane reservoir to achieve the measured $\mathrm{D} / \mathrm{H}$ ratio.

There is nothing to distinguish among the curves in Fig. 4 in and of themselves. However, two constraints are available. The weaker constraint is that of the initial $\mathrm{D} / \mathrm{H}$ ratio in the material out of which Titan was formed. Deuterium enrichment in organic molecules in giant molecular clouds is very large; $\mathrm{DCN} / \mathrm{HCN}$ for example is greater than the protosolar value by one to two orders of magnitude (Van Dishoeck et al., 1993). In Comet Hale-Bopp DCN/HCN is about 150 times the protosolar value (Meier et al., 1998a). $\mathrm{HDO} / \mathrm{H}_{2} \mathrm{O}$ as measured in Halley, Hale-Bopp and Hyakutake is about 20 times the protosolar value (Bockelée-Morvan et al., 1998; Meier et al., 1998b; Balsiger et al., 1995; Eberhardt et al., 1995). Thus, unless other comets (for example, from the Kuiper Belt rather than the Oort Cloud) show much smaller $\mathrm{D} / \mathrm{H}$ enrichments, condensed matter from the outer solar nebula would seem to be a poor direct source for Titan's volatiles. A circum-Saturnian nebula would be warmer and exhibit more processing of volatiles than the surrounding solar nebula; rough estimates for simple models of 


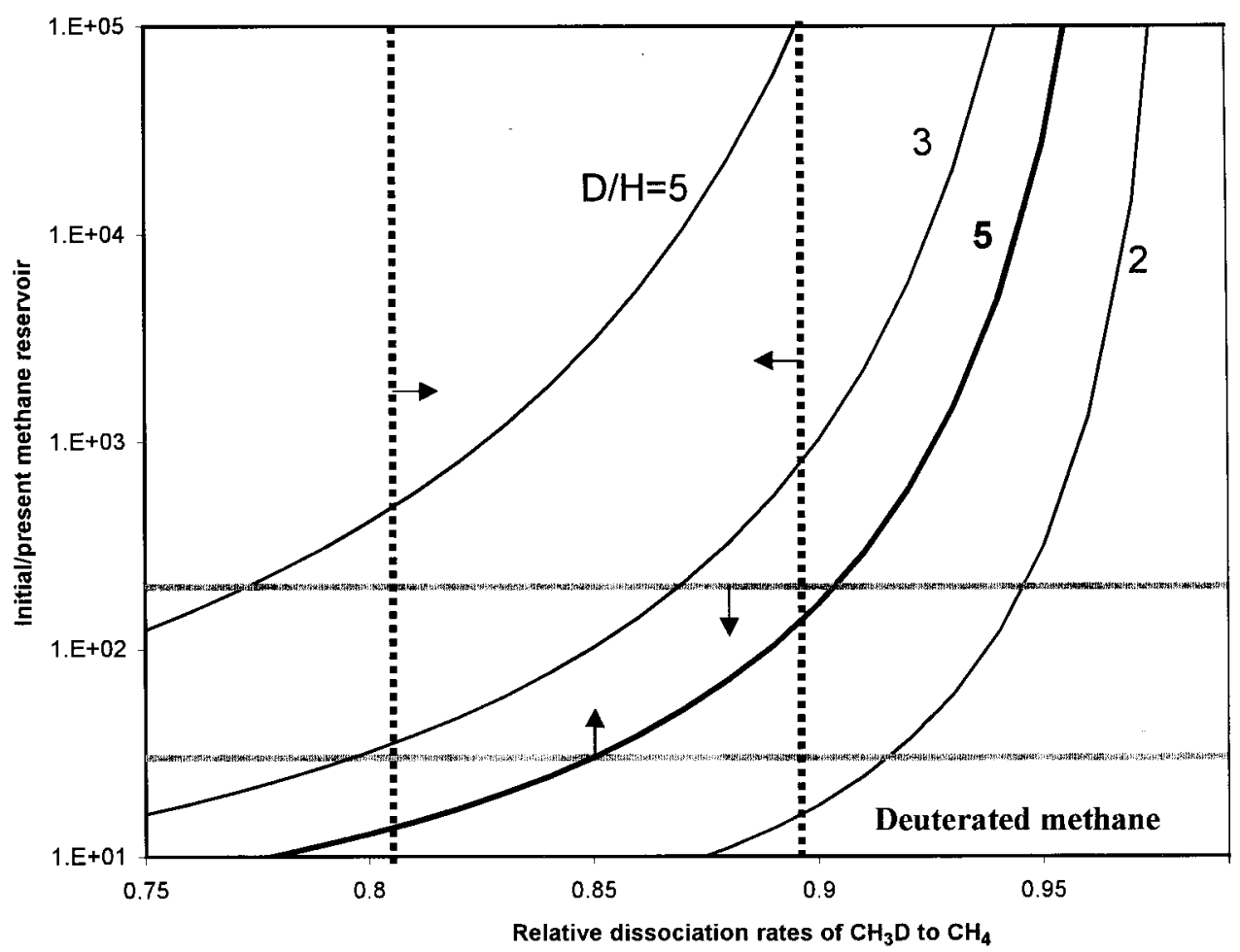

Fig. 4. The fractionation of deuterium in methane photochemistry. Plotted is the initial mass of the methane reservoir, normalized to the mass of the current reservoir, as a function of the relative dissociation rates of deuterated and normal methane (the $q$ factor defined in the text). Three curves are shown corresponding to different present-day $\mathrm{D} / \mathrm{H}$ ratios in methane, relative to the protosolar $\mathrm{D} / \mathrm{H}$ abundance, as labelled. The starting $\mathrm{D} / \mathrm{H}$ ratio, that in the planetesimals from which Titan formed, is assumed to be 1.5 times the protosolar value for the thin curves. The heavy curve assumes a starting $\mathrm{D} / \mathrm{H}=3$ times the protosolar value. The horizontal lines show the limits on the initial methane reservoir based on the assumption of constant photolysis of methane on Titan over the age of the solar system. The vertical lines represent limits on plausible values of $q$.

such a disk give $\mathrm{CH}_{3} \mathrm{D} / \mathrm{CH}_{4}$ approximately 1 to 2 times the protosolar value (Lunine and Tittemore, 1993). We regard this constraint as a weak one until more detailed nebular models are available.

The second constraint comes from the bulk photolysis of methane itself. Voyager 1 data, combined with photochemical models, yield a current photolysis rate that is well-determined to within a factor of two (Yung et al., 1984; Lara et al, 1994; Toublanc et al., 1995). Although the published models differ in the proportions of the various products of methane photolysis, there is close agreement in total destruction rate of methane: photolysis is limited by the solar ultraviolet flux with energies above the threshold for methane dissociation. Thus under the assumption of a steadystate, which here means the constant availability of methane in Titan's atmosphere, we know to within an order of magnitude how much has been destroyed over the age of the solar system. Then the ratio $R$ of the current atmospheric mass of methane to the total destroyed over the age of the solar system (which could include a surface condensed component) is approximately
$R=P_{m} / g m F \Delta t$

where $P_{m}$ is the partial pressure of methane, $g$ the gravity, $F$ the destruction rate in molecules per unit area (all parameters referring or corrected to the surface), $m$ the mass of a methane molecule and $\Delta t$ the age of the solar system. Uncertainties in Eq. (11) are the photolysis rate and the partial pressure of atmospheric methane near surface (which dominates the mass); the latter is uncertain to a factor of 4 .

Corresponding limits are given in Fig. 4 by the horizontal lines; we have not taken an additional spread for uncertainties in the individual photochemical models because we regard the differences among the models to be sufficiently representative of such uncertainties. Further, the lower limit is consistent with the lack of remote sensing evidence for global photochemical debris since it is equivalent to a depth of detritus that could be hidden in crater basins at high latitudes (Lorenz et al., in prep.). Significantly smaller values on the $y$-axis of Fig. 4 are possible under the assumption that photolysis of methane is intermittent, which in turn could mean that the current atmosphere is not typical of that over most of the satellite's history (Lorenz et al., 1997). 
The limits on the initial methane reservoir from bulk methane photochemistry are essentially independent of the limits from the deuterium abundance, except for the underlying mechanism of photolytic breakup of methane followed by hydrogen loss. Thus, the two approaches could have yielded vastly different initial reservoirs in Fig. 4. In fact, consistency in the initial reservoir size is achieved, within the error bars for the measured $\mathrm{D} / \mathrm{H}$ ratio, under the assumption of a primordial deuterium enrichment of 1 to 3 times the protosolar value and the relative dissociation rates $q$ for $\mathrm{CH}_{3} \mathrm{D}$ versus $\mathrm{CH}_{4}$ calculated above.

\section{Implications}

\subsection{Deuterated methane and the source region for Titan's volatiles}

The ratio $\mathrm{CH}_{3} \mathrm{D} / \mathrm{CH}_{4}$ in Titan's atmosphere is most simply and directly interpreted to be the result of photochemical enrichment of deuterium through that isotope's preferential retention during methane photolysis, based on the calculations summarized in Fig. 4. Photolytic enrichment limits the amount of fractionation Titan's methane could have initially possessed to at most a few times the protosolar value. This, in turn, appears to rule out the atmospheric source as being derived from Oort Cloud comets such as Hale-Bopp, Hyakutake, and Halley - all of which have very strong deuterium enrichments. In particular, a model (Griffith and Zahnle, 1995) that relies on impacting comets as the source is difficult to reconcile with the state of Titan's atmospheric deuterium.

An alternative is that Titan derived its volatile inventory, including methane, from a region around Saturn strongly altered by that giant planet's formation. This region possessed elevated gas pressures and temperatures compared to the surrounding solar nebula (Magni and Coradini, 1999). Gases processed at higher temperatures in the inner part of the disk could become trapped in ices farther out, and then were accreted into the growing satellites including Titan. A key issue is how much mixing might have occurred in such a disk. Simple considerations, including the compact scale of the disk and high luminosity, suggest rather efficient mixing: hence much or all of the gases trapped in the ice experienced high temperatures at some point. Based on such a picture Lunine and Tittemore (1993) estimated that the $\mathrm{D} / \mathrm{H}$ ratio in ices in the circum-Saturnian disk should be limited to about 1.5 times the protosolar value. As noted above, this is consistent with the results in Fig. 4. More detailed disk models in the near future will allow this number to be calculated in greater detail, although the usual uncertainties in such models will limit the extent to which the enrichment can be constrained with certainty.

\subsection{Titan's early history based on the enrichments of ${ }^{15} \mathrm{~N}$ and $\mathrm{CH}_{3} \mathrm{D}$}

A key issue is whether an epoch of rapid atmospheric escape of nitrogen, invoked to enhance the heavy nitrogen abundance, would also alter the deuterated methane abundance. Indeed $\Delta m=1$ for both sets of isotopes, and so we expect deuterium to be enhanced along with heavy nitrogen - either through preferential loss of $\mathrm{CH}_{4}$ or (much more likely) $\mathrm{H}$ itself. Reference to Fig. 2 shows that many tens or even hundreds of times the current mass of atmospheric nitrogen must be lost to achieve the current nitrogen isotopic enrichment above the primordial value. If methane were similarly enhanced during an era of early escape it would produce the current $\mathrm{CH}_{3} \mathrm{D} / \mathrm{CH}_{4}$ ratio without any photochemical enrichment. Reference to Fig. 4 then shows that essentially little or no further enrichment by photolysis could be tolerated.

How do we reconcile this picture with the apparent reasonableness and internal consistencies of the separate stories for the two isotopes? There are two possibilities. First, it is conceivable that the $q$ value expressing the relative dissociation rates of $\mathrm{CH}_{3} \mathrm{D}$ and $\mathrm{CH}_{4}$ might be 0.9 or greater - that is, deuterium is more readily removed by photolysis than we asserted earlier. However, this seems inconsistent with the laboratory studies and simple theoretical considerations having to do with the relative bonding energies of $\mathrm{D}$ and $\mathrm{H}$ in methane as described above. A second possibility, examination of which occupies the remainder of this section, is that significant amounts of methane were outgassed from Titan's interior after an early epoch of atmospheric loss enriched the nitrogen isotopes.

Interior models of Titan predict a substantial liquid ammonia-water layer surviving to the present day if ammonia was included in the initial inventory of volatiles (Lunine and Stevenson, 1987). Methane is soluble enough in the liquid that substantial quantities could be stored in the interior indefinitely. Even in the absence of such an ammonia-water liquid mantle, methane can be stored in the water ice as clathrate hydrate up to pressures of at least 5 kilobars (Lunine and Stevenson, 1985). Molecular nitrogen is not nearly as soluble in water-ammonia liquid or in the clathrate hydrate. A plausible story, then, that could lead to the present isotopic situation is as follows:

1. Titan accretes large amounts of nitrogen (as molecular nitrogen or ammonia) and carbon (as methane). A primordial atmosphere of ammonia, or molecular nitrogen with some methane, undergoes 
rapid escape and enrichment of the heavier isotopes. If the nitrogen is in the form of ammonia, photochemical or impact-generated chemistry converts that molecule to molecular nitrogen (Atreya et al., 1978; McKay et al., 1988; Jones and Lewis, 1987; Atreya et al., 1978). As the atmosphere and surface cool down, the residual ammonia (much less volatile than the nitrogen) condenses on the surface leaving the molecular nitrogen behind in the gas phase.

2. The interior of Titan after accretion is a mixed icerock core overlain by rock and then water ice, the result of accretional heating that increased as the mass of the growing planet increased (Stevenson et al., 1986). As radioisotopic heating builds, the ice in the homogeneous core softens and initiates a core overturn which moves softened ice upward, rock downward, and in consequence releases gravitational energy. Melting of the overlying water ice and heating of any ammonia-water mantle release volatiles from the interior to the surface. By virtue of their relative propensities for being trapped in the icy planetesimals from which Titan formed, methane is much more abundant in the outgassed products than is molecular nitrogen. Hence, the outgassing primarily releases methane into the surfaceatmosphere system. This core-forming event, coming a few hundred million years after accretion, is subsequent to the era of escape-driven fractionation of the primordial atmosphere. The methane released dominates over any methane left from the primordial atmosphere.

3. The newly outgassed methane-depending on its abundance-saturates in the atmosphere, partially condenses onto the surface and collects into the immediate subsurface. Any cryovolcanic flows associated with the core overturn do not put significant amounts of ammonia into the atmosphere relative to methane, because of the vast difference in volatility of the two materials. The result is an atmosphere containing the isotopically fractionated molecular nitrogen from early escape (and production of $\mathrm{N}_{2}$ from $\mathrm{NH}_{3}$ ), unfractionated methane from the later core-forming event, and a fresh crust of water ice possibly mixed with ammonia flows. This crust hosts, atop it or within it, some amount of the outgassed methane that could not be accommodated by the cooling atmosphere.

This model is cosmochemically sound and produces a volatile inventory after the first $10 \%$ of Titan's history that allows the current isotopic fractionations of nitrogen and hydrogen to be understood. It is also consistent with the carbon isotopic ratios ${ }^{13} \mathrm{C} /{ }^{12} \mathrm{C}$ in $\mathrm{HCN}$ and in $\mathrm{C}_{2} \mathrm{H}_{6}$ that are not enhanced relative to the terrestrial value. These ratios reflect a bulk carbon inventory (i.e., principally methane) that did not participate in the massive escape episode of the post-accretional atmosphere that was largely nitrogen, ammonia, or both.

The model implies that icy bodies derived from the solar nebula (i.e., comets) did not supply Titan's volatile inventory. The deuterium abundance on Titan by itself argues against such a supply. Instead, relatively deuterium-poor methane from a proto-Saturnian disk of material is the source. Whether such a disk would supply nitrogen in the form of ammonia or molecular nitrogen remains unclear until detailed thermochemical and kinetics calculations can be conducted. But in either case, the nitrogen isotopes fractionate first by escape, and the deuterium carried in methane is then slowly fractionated by photochemistry over the next 4 billion years or so.

This model for the origin and early evolution of volatiles on Titan can be subjected to multiple tests, not the least of which is improved determination of the nitrogen, carbon, and hydrogen isotopic abundances in Titan's atmosphere. It is important to be able to measure the isotopic ratio in $\mathrm{N}_{2}$ itself, as this is the principal nitrogen gas, but such a measurement must await the Huygens probe descent in 2004. Measurement of the carbon isotopes in methane in Titan's atmosphere would provide a slightly more direct constraint on whether methane itself participated in the early escape episode that fractionated the nitrogen. Determination of the argon isotopes is also important for assessing the amount of ammonia-water outflow over Titan's history (Engel et al., 1994), and, finding evidence for a significant thermal event driven by core overturn. Noble gas abundances will also constrain from a different standpoint the composition of the ices from which Titan was formed, and whether nitrogen was added as ammonia or molecular nitrogen (Owen, 1982). Improved understanding of the present upper atmospheric thermal and density structure from more detailed ultraviolet observations will provide a contemporary baseline for escape models. The Huygens Probe or Cassini Orbiter could perform all of the required observations during the prime mission phase beginning in 2004. Equally important is to conduct laboratory measurements to verify and tighten the range of $q$ derived here.

The ultimate utility of understanding the origin of the isotopic fractionations on Titan is in constraining how large bodies formed around the giant planets. The Galilean moons and Titan were clearly major events in the processes leading to the giant planet systems we see today. Along with tying down the details of outer solar system formation, understanding how and where such giant moons come from has an implication for the existence, frequency and nature of large natural satellites around newly discovered extra-solar giant planets. Since some of these 'Jupiters' orbit at modest 
distances from the parent stars, their putative giant moons might well serve as hosts for life-provided they retain the sorts of volatiles that Titan has in abundance in the present-day.

\section{Acknowledgements}

The first author is most grateful to Dr Angioletta Coradini for hosting his sabbatical at the Istituto di Astrofisica Spaziale, Rome, during the preparation of this paper. Helpful and substantive referee suggestions, as well as those of Dr Daniel Gautier, improved the quality of the work. Support for the project and paper were provided by the Consiglio Nazionale della Ricerche and NASA's Atmospheres and Geoscience programs.

\section{References}

Atreya, S.K., Donahue, T.M., Kuhn, W.R., 1978. Evolution of a nitrogen atmosphere on Titan. Science 201, 611-613.

Balsiger, H., Altwegg, K., Geiss, J., 1995. D/H and ${ }^{18} \mathrm{O} /{ }^{16} \mathrm{O}$ ratio in the hydronium ion and in neutral water from in situ ion measurements in Comet P/Halley. J. Geophys. Res. 100, 5834.

Bockelée-Morvan, D., Gautier, D., Lis, D.C., Young, K., Keene, J., Phillips, T.G., Owen, T., Crovisier, J., Goldsmith, P.F., Bergin, E.A., Despois, D., Wooten, A., 1998. Deuterated water in comet C/1996 B2 (Hyakutake) and its implications for the origin of comets. Icarus 133, 147-162.

Cosby, P.C., 1993. Electron-impact dissociation of nitrogen. J. Chem. Phys. 98, 9544-9553.

Courtin, R., Gautier, D., McKay, C.P., 1995. Titan's thermal emission spectrum: reanalysis of the Voyager infrared measurements. Icarus 114, 144-162.

Coustenis, A., Bezard, B., Gautier, D., 1989. Titan's atmosphere from Voyager infrared observations. II. The $\mathrm{CH}_{3} \mathrm{D}$ abundance and $\mathrm{D} / \mathrm{H}$ ratio from the $900-1200 \mathrm{~cm}^{-1}$ spectral region. Icarus $82,67-180$.

De Bergh, C., Lutz, B.L., Owen, T., Chauville, J., 1988. Monodeuterated methane in the outer solar system. III. Its abundance on Titan. Astrophys. J. 329, 951-955.

DeMore, W.B. et al., 1997. Chemical kinetics and photochemical data for use in stratospheric modeling, evaluation No. 11. JPL Publ. 97-4 (Generic).

Eberhardt, P., Reber, M., Krankowski, D., Hodges, R.R., 1995. The $\mathrm{D} / \mathrm{H}$ and ${ }^{18} \mathrm{O} /{ }^{16} \mathrm{O}$ ratios in water from Comet $\mathrm{P} / \mathrm{Halley}$. Astron. Astrophys. 302, 301-316.

Engel, S., Lunine, J.I., Norton, D.L., 1994. Silicate interactions with ammonia-water fluids on early Titan. J. Geophys. Res. 99, 37453752.

Fegley, B. Jr, 1997. Analysis of Returned Comet Nucleus Samples In: Chang, S. (Ed.), NASA CP-10152, Washington, D.C., pp. 7391.

Fox, J.L., Hac, A., 1997. The ${ }^{15} \mathrm{~N} /{ }^{14} \mathrm{~N}$ isotope fractionation in dissociative recombination of $\mathrm{N}_{2}^{+}$. Journal of Geophysical Research 102, 9191-9204.

Gautier, D., 1997. The aeronomy of Titan, In: Lebreton, J.P. (Ed.), Huygens: Science, Payload and Mission. ESA SP-1177, ESA Publications Department, ESTEC, Noordwijk, The Netherlands, pp. 199-209.

Geiss, J., Gloeckler, J., 1998 Abundances of deuterium and helium 3 in the protosolar cloud from solar wind measurements, Space Science Rev. 84, 239-250.

Gierczak, T., Talukdar, R.K., Herndon, S.C., Vaghjiani, G.L., Ravinshankara, A.R., 1997. Rate coefficients for the reactions of hydroxyl radicals with methane and deuterated methanes. J. Physical Chemistry A 101, 3125-3134.

Griffith, C., Zahnle, K., 1995. Influx of cometary volatiles to planetary moons: the atmospheres of 1000 possible Titans. J. Geophys. Res. 100, 16,907-16,922.

Hidayat, T., Marten, A., 1998. Evidence for a strong ${ }^{15} \mathrm{~N} /{ }^{14} \mathrm{~N}$ enrichment in Titan's atmosphere from millimeter observations. Annales Geophysicae 16 (Suppl. III) C998 (Abstract).

Hidayat, T., Marten, A., Bezard, B., Gautier, D., Owen, T., 1997. Millimeter and submillimeter heterodyne observations of Titan: retrieval of the vertical profile of $\mathrm{HCN}$ and the ${ }^{12} \mathrm{C} /{ }^{13} \mathrm{C}$ ratio. Icarus 126, 170-182.

Hunten, D.M., Donahue, T.M., Walker, J.C.G., Kasting, J.F., 1989. In: Atreya, S.K., Pollack, J.B., Matthews, M.S. (Eds.), Origin and Evolution of Planetary and Satellite Atmospheres. University of Arizona Press, Tucson, pp. 386-422.

Jones, T.D., Lewis, J.S., 1987. Estimated impact shock production of $\mathrm{N}_{2}$ and organic compounds on early Titan. Icarus 72, 381-393.

Lammer, H., Bauer, S.J., 1991. Nonthermal atmospheric escape from Mars and Titan. Journal of Geophysical Research 96, 18191825.

Lammer, H., Bauer, S.J., 1993. Atmospheric mass loss from Titan by sputtering. Planetary and Space Science 41, 657-663.

Lander, D.R., Unfried, K.G., Glass, G.P., Curl, R.F., 1990. Rateconstant measurements of $\mathrm{C}_{2} \mathrm{H}$ with $\mathrm{CH}_{4}, \mathrm{C}_{2} \mathrm{H}_{6}, \mathrm{C}_{2} \mathrm{H}_{4}, \mathrm{D}_{2}, \mathrm{CO}$. J. Physical Chemistry 94, 7759-7763.

Lara, L.M., Lorenz, R.D., Rodrigo, R., 1994. Liquids and solids on the surface of Titan. Planetary and Space Science 42, 5-14.

Laufer, A., 1981. Reactions of ethynyl radicals. Rate constants with $\mathrm{CH}_{4}, \mathrm{C}_{2} \mathrm{H}_{6}$, and $\mathrm{C}_{2} \mathrm{D}_{6}$. J. Physical Chemistry 85, 3828-3831.

Lecluse, C., Robert, F., Gautier, D., Guiraud, M., 1996. Deuterium enrichment in giant planets. Planetary and Space Science 44, $1579-1592$.

Lorenz, R.D., Lunine, J.I., 1997. Titan's surface reviewed: the nature of bright and dark terrain. Planetary and Space Science 45, 981992.

Lorenz, R.D., McKay, C.P., Lunine, J.I., 1997. Photochemicallyinduced collapse of Titan's atmosphere. Science 275, 642-644.

Lunine, J.I., 1985. Volatiles in the outer solar system. Dissertation, p. 326.

Lunine, J.I., Owen, T.C., Brown, R.H., 2000. In: Mannings, V., Boss, A.P., Russel, S. (Eds.), Protostars and Planets IV. University of Arizona Press, Tucson (in press).

Lunine, J.I., Stevenson, D.J., 1985. Thermodynamics of clathrate hydrate at low and high pressures with application to the outer solar system. Astrophys. J. Suppl. Ser. 58, 493-531.

Lunine, J.I., Stevenson, D.J., 1987. Clathrate and ammonia hydrates at high pressure: application to the origin of methane on Titan. Icarus 70, 61-77.

Lunine, J.I., Tittemore, W.B., 1993. In: Levy, E.H., Lunine, J.I. (Eds.), Protostars and Planets III. University of Arizona Press, Tucson, pp. 1149-1176.

Magni, G., Coradini, A., 1999 The formation of Saturn: primordial phases, Planetary and Space Science. Submitted.

Mahaffy, P.R., Donahue, T.M., Atreya, S.K., Owen, T.C., Niemann, H.B., 1998. Galileo probe measurements of $\mathrm{D} / \mathrm{H}$ and ${ }^{3} \mathrm{He} /{ }^{4} \mathrm{He}$ in Jupiter's atmosphere. Space Science Reviews 84, 251-263.

Marten, A., Hidayat, T., Bezard, B., Gautier, D., Owen, T., 1997. IAU Circular 6702 (19 June 1997).

McKay, C.P., Scattergood, T.W., Pollack, J.B., Borucki, W.J., Van Ghysegahm, H.T., 1988. High temperature shock formation of $\mathrm{N}_{2}$ and organics on primordial Titan. Nature 332, 520-522.

Meier, R., Owen, T.C., Jewitt, D.C., Matthews, H.E., Senay, M., 
Biver, N., Bockelée-Morvan, D., Crovisier, J., Gautier, D., 1998a. Deuterium in comet C/1995 O1 (Hale-Bopp): detection of DCN. Science 279, 1707-1710.

Meier, R., Owen, T.C., Matthews, H.E., Jewitt, D.C., BockeléeMorvan, D., Biver, N., Crovisier, J., Gautier, D., 1998b. A determination of the $\mathrm{HDO} / \mathrm{H}_{2} \mathrm{O}$ ratio in comet $\mathrm{C} / 1995 \mathrm{O} 1$ (HaleBopp). Science 279, 842-844.

Mumma, M.J., 1997. In: Pendleton, Y.J., Tielens, A.G.G.M. (Eds.). From Stardust to Planetesimals ASP Conference Series, San Francisco, pp. 369-396.

Orton, G.S., 1992. In: Symposium on Titan. ESA SP-338, Noordwijk, Netherlands, pp. 81-85.

Ott, U., 1993. In: Levy, E.H., Lunine, J.I. (Eds.), Protostars and Planets III. University of Arizona Press, Tucson, pp. 883-902.

Owen, T.C., 1982. The composition and origin of Titan's atmosphere. Planetary and Space Science 30, 833-838.

Pinto, J.P., Lunine, J.I., Kim, S-J., Yung, Y.L., 1986. D to H ratio and the origin and evolution of Titan's atmosphere. Nature 319, 388-390.

Renlund, A.M., Shokoohi, F., Reisler, H., Wittig, C., 1982. Reaction of $\mathrm{C}_{2} \mathrm{H}$ with $\mathrm{O}_{2}$ : chemiluminescent products. J. Physical Chemistry 86, 4165-4170.

Sagan, C., Thompson, W.R., 1984. Production and condensation of organic gases in the atmosphere of Titan. Icarus 59, 133-161.

Smith, P.H., Lemmon, M.T., Lorenz, R.D., Sromovsky, L.A.,
Caldwell, J.J., Allison, M.D., 1996. Titan's surface, revealed by HST imaging. Icarus 119, 336-339.

Stevenson, D.J., 1992. Proceedings of the Symposium on Titan. ESA SP-338, Noordwijk, The Netherlands, pp. 29-33.

Stevenson, D.J., Harris, A.W., Lunine, J.I., 1986. In: Burns, J.A., Matthews, M.S. (Eds.), Satellites. University of Arizona Press, Tucson, pp. 39-88.

Strobel, D.F., Summers, M.E., Zhu, X., 1992. Titan's upper atmosphere: structure and ultraviolet emissions. Icarus 100, 512-526.

Toublanc, D., Parisot, J.P., Brillet, J., Gautier, D., Raulin, F., McKay, C.P., 1995. Photochemical modeling of Titan's atmosphere. Icarus 113, 2-26.

Van Dishoeck, E.F., Blake, G.A., Draine, B.T., Lunine, J.I., 1993. In: Levy, E.H., Lunine, J.I. (Eds.), Protostars and Planets III. University of Arizona Press, Tucson, pp. 163-241.

Vervack, R., 1997. Titan's upper atmospheric structure derived from Voyager ultraviolet spectrometer observations. Dissertation, University of Arizona, Tucson.

Wallington, T.J., Hurley, M.D., 1992. Atoms with $\mathrm{CF}_{3} \mathrm{CHC}_{12}$, $\mathrm{CF}_{3} \mathrm{CH}_{2} \mathrm{~F}, \mathrm{CFC}_{12} \mathrm{CH}_{3}, \mathrm{CF}_{2} \mathrm{ClCH}_{3}, \mathrm{CHF}_{2} \mathrm{CH}_{3}, \mathrm{CH}_{3} \mathrm{D}, \mathrm{CH}_{2} \mathrm{D}_{2}$, $\mathrm{CHD}_{3}, \mathrm{CD}_{4}, \mathrm{CD}_{3} \mathrm{Cl}$ at $295 \pm 2 \mathrm{~K}$. Chemical Physics Letters 189 , 437-442.

Yung, Y.L., Allen, M.A., Pinto, J.P., 1984. Photochemistry of the atmosphere of Titan: comparison between model and observations. Astrophys. J. Suppl. Ser. 55, 465-506. 